\title{
Control of a Battery Energy Storage System Accounting for the Charge Redistribution Effect to Dispatch the Operation of a Medium Voltage Feeder
}

\author{
Fabrizio Sossan, Dimitri Torregrossa, Emil Namor, Rachid Cherkaoui, Mario Paolone \\ Distributed Electrical Systems Laboratory (DESL) \\ EPFL, École Polytechnique Fédérale de Lausanne, Switzerland \\ \{fabrizio.sossan, dimitri.torregrossa, emil.namor, rachid.cherkaoui, mario.paolone\}@epfl.ch
}

\begin{abstract}
We describe a process to dispatch the operation of a medium voltage distribution feeder and improve its qualityof-service using a battery energy storage system (BESS). It is organized according to a day-ahead and intra-day structure to allow the integration with electricity markets. In the former stage, the average power transit on a 5-minute resolution is determined for the next day of operation using adaptive blackbox forecasting. In the latter stage, the feeder operation is dispatched according to the previously determined trajectory. The resulting tracking problem is accomplished by using the BESS to compensate for deviations from the dispatched plan, which are likely to occur due to forecasting errors. For the first time in the literature, the control strategy is realized using model predictive control (MPC) accounting for the battery charge redistribution effect. This phenomena is described using a stateof-the-art lithium battery model. A simulated proof-of-concept is given.
\end{abstract}

Index Terms-Batteries, Energy management, Optimization

\section{INTRODUCTION}

The progressive displacement of conventional generation in favor of renewables requires to reallocate an adequate level of controllability to assure reliable power system operations. Additionally, the intrinsic dispersed nature of renewable sources poses quality-of-service (QOS) issues, such as increased voltage variations along the feeders, that cannot be effectively handled with the current power system architecture, since it was designed for unidirectional powers flow and centralized control. Decentralizing and extending the control infrastructure to the distribution level is also of interest in the case of grid congestion management, which is often considered as a viable alternative to otherwise expensive distribution networks reinforcements. An emerging mainstream concept to face the aforementioned challenges is achieving the controllability of portions of distribution networks to satisfy assigned trajectories of the active and reactive power transits thanks to, e.g., deploying battery energy storage systems (BESSs) and demand response (DR) programs. This paradigm can be traced in a number of frameworks, such as virtual power plants

Financial support by $i$ ) Canton of Vaud within the framework of the "100 millions pour les énergies renouvelables et l'efficacité énergétique" program and ii) SNSF - NRP 70 "Energy Turnaround".
(VPPs) and microgrids. The former consists of aggregating several units, possibly of different kinds, to accomplish a given common target, such as dispatchable power and ancillary services to the upper-grid layer. The latter framework is similar to a technical VPP (where units are aggregated by topology, instead of being grouped in portfolios, as in commercial VPP) with the difference that microgrids can operate independently and disconnected from the main grid. Both setups have advanced monitoring and ICT requirements (e.g. [1]), which are not met within the current power system framework. If the transition to a smarter grid has to happen, it will be likely characterized by step-by-step progresses rather than a sudden revolution; it is hence of importance to research on plug and play methods, i.e. solutions that can operate in the existing framework without requiring deep changes to the overall power system infrastructure and development of new policies. With this requirement in mind, in this paper we investigate on how to achieve dispatchability of a distribution feeder. The proposed configuration utilizes a BESS, MPC and adaptive data-driven consumption forecasting and is characterized by a low overall complexity because the decision process only requires locally available information, and all the components can be conveniently placed in one location, such as in a secondary substation. In the literature, BESSs control strategies were mainly proposed in connection to smoothing, storing and scheduling of DERs production, and managing network congestions [2]-[7]. We extend the existing literature by proposing to dispatch the operation of a distribution feeder using, for the first time in literature, a MPC algorithm that accounts for the battery charge redistribution effect through a state-of-the-art battery model.

\section{Problem statement}

\section{A. Aims and motivations}

We consider a generic distribution feeder populated by an unknown mix of electric loads and possibly distributed generation (DG). The load and DG power injections are unknown, while the power transit at the substation transformer is measured. The idea is to utilize a BESS to accomplish the following two objectives: 
1) dispatching the operation of the distribution feeder, i.e. the power consumption of the feeder should stick to a trajectory that is determined the day before the operation. This is to reduce the stochasticity associated to the power consumption, hence allowing for decreasing the amount of regulating power required to operate the grid, a key issue especially with large shares of production from renewables;

2) clearing potential congestions of the substation transformer in order to respect its rating and keep adequate voltage level along the feeder, allowing for a reliable delivery of the electricity.

\section{B. Network setup and requirements}

The configuration of the network is sketched in Fig. 1. The BESS, in the bottom right corner and composed by the battery and the respective management system (BMS), is located at the root of the feeder and is operated by the DSO. The monitoring and control requirements (top left corner) consist uniquely in measuring the power consumption transit at the secondary substation, controlling the BESS power injection and bidirectional communication with the DSO control room. The feeder on the right part of Fig. 1 does not require any infrastructure deployment or development (compare with demand response programs which requires extended control and monitoring infrastructures).

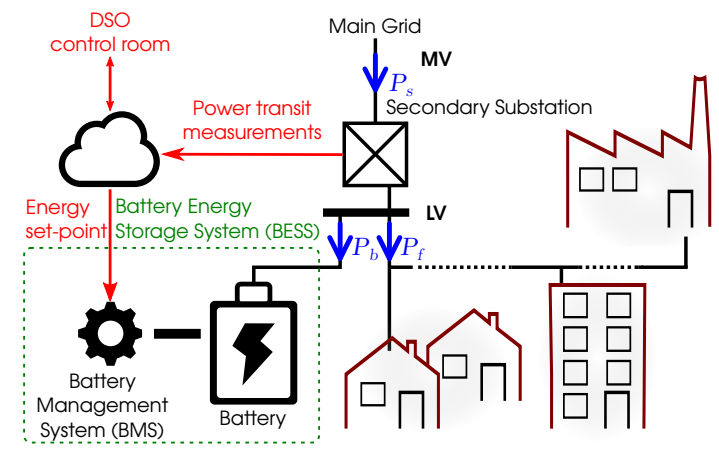

Fig. 1. The configuration of the dispatchable SOCdistribution feeder. The control and monitoring infrastructure is shown in red, and, in blue, the power transit measurements.

\section{METHODS}

To facilitate the possible future integration with liberalized electricity markets, the proposed framework is similarly organized according to a day-ahead of and intra-day operation structure. Briefly, in the former stage, the 5-minute power consumption trajectory of the feeder for the next day of operation, said advertised power consumption, is determined. A 5-minute period is chosen since it is a common choice for real time electricity markets [8]. In the latter stage, the BESS is utilized to compensate for mismatches between the advertised power consumption and realizations, which are likely to occur because forecast errors. Equivalently, we can say that during operation the BESS is utilized to track the advertised power consumption. A sketch of the communication happenings are shown in Fig. 2.

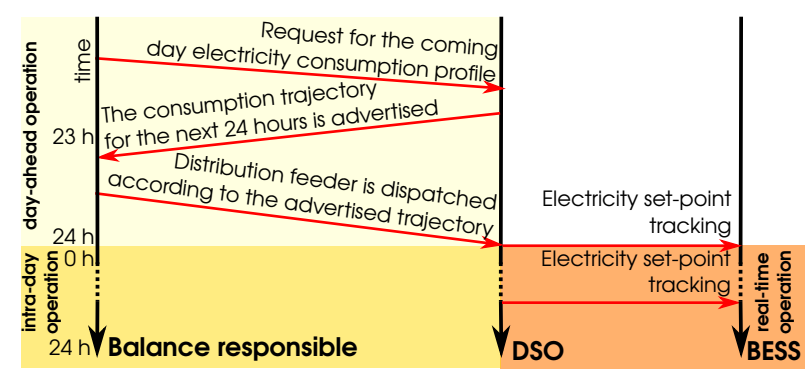

Fig. 2. Message exchange between a balance responsible (e.g. TSO), DSO and the BESS for the operation of the dispatchable feeder.

\section{A. Day-ahead of operation}

This phase takes place several hours ahead the day of operation. In a real operation context, it should be completed in time to be integrated within day-ahead market operation. In fact, scheduling the operation of distribution feeders could impact market decisions as the stochasticity of the consumption forecast, as well as the risk of incurring in unbalances, is reduced. In this paper, we arbitrarily chose to perform the operation described hereafter by 11PM of the day before operation. The objective of this phase is to obtain the advertised power consumption trajectory $\left[P_{0}^{*}, \ldots, P_{N-1}^{*}\right]$, namely the sequence of 5-minute active power average values to which the substation power transit should stick to during the next day. For each 5 -minute interval $t=0, \ldots, N-1$ of the next day (where $N=120$ is the number of 5-minute interval in 24 hours), the advertised power consumption is as:

$$
P_{t}^{*}=\widehat{L}_{t}+\widehat{B}_{t}^{o}
$$

where $\widehat{L}_{t}$ and $\widehat{B}_{t}^{o}$ respectively denote the power consumption forecast and battery charging demand. In words, we say that the substation power transit prediction is given by the next day load forecasting and the demand necessary to bring the battery state of charge (SOC) to $50 \%$ during the first hours of the day of operation (further details are in Section III-A2).

1) Day-ahead power consumption forecasting: The feeder day-ahead power consumption forecast are obtained using an adaptive data-driven black-box forecasting method based on vector autoregression (VAR). This choice is because black-box modelling does not rely on any assumptions on the nature of the electric loads to model (cfr. grey-box modelling). Thus, it is generic enough to be applied for the considered case, where the composition of the feeder in terms of number and kinds of electric loads is unknown. The power consumption forecast for the day $d+1$ is denoted by the column vector $\widehat{\mathcal{L}}_{d+1 \mid d}=$ $\left[\widehat{L}_{0}, \ldots, \widehat{L}_{N-1}\right]$ and determined as a linear combination of the historic power consumption sequences with the same day of week (Monday, Sunday ...) as $d+1$. Alternatively, we say that the power consumption of, for example, a Thursday is a weighted average of the given power consumption sequences 
of past Thursdays. Hence, it can be expressed as:

$$
\widehat{\mathcal{L}}_{d+1 \mid d}=a_{1} \mathcal{L}_{d+1-7}+\cdots+a_{p} \mathcal{L}_{d+1-7(p+1)},
$$

where $p$ and $a_{1}, \ldots, a_{p}$ are respectively the autoregressive term degree and coefficients. The sequences $\mathcal{L}_{(d+1)-7}, \ldots, \mathcal{L}_{(d+1)-(p+1) \cdot 7}$ are obtained by splitting the historical feeder power consumption measurements into 1day long sequences. At this stage, both the degree and the coefficients of the VAR model in (2) are empirically chosen as:

$$
\begin{aligned}
& p=5 \\
& a_{i}=\frac{1}{p}, \quad i=1, \cdots, p .
\end{aligned}
$$

The uncertainty associated to each point prediction in $\widehat{\mathcal{L}}_{d+1 \mid d}$ is estimated by the variance $\hat{\mathcal{V}}_{d+1 \mid d}=\left[\widehat{V}_{0}, \ldots, \widehat{V}_{N-1}\right]$ of the respective regressors. Hence, the following relationship holds:

$$
\begin{aligned}
& \hat{\mathcal{V}}_{d+1 \mid d}= \\
& =\frac{1}{p} \sum_{i=0}^{p-1} \operatorname{diag}\left(\overline{\mathcal{L}}_{d}-\mathcal{L}_{d+1-7(i+1)}\right)\left(\bar{L}_{d}-\mathcal{L}_{d+1-7(i+1)}\right)
\end{aligned}
$$

where the column vector $\overline{\mathcal{L}}_{d}$ denotes the average of the vector regressors, i.e. :

$$
\overline{\mathcal{L}}_{d}=\frac{1}{p} \sum_{i=0}^{p-1} \mathcal{L}_{d+1-7(i+1)} .
$$

2) Day-ahead battery charging demand: As it will be shown in the following section, during intra-day operation, the battery is utilized to compensate for the deviations from the advertised power consumption profile in order to achieve an energy balance control. Hence, its SOC might deviate substantially from 0.5 , i.e. the ideal value to compensate for an unbiased (i.e. with zero mean errors) advertised power consumption profile. The following optimization problem determines the optimal current trajectory $\boldsymbol{i}^{o}=\left[i_{0}^{o}, \ldots, i_{N-1}^{o}\right]$ to achieve the 0.5 SOC starting from the SOC at time $t=0$ (0AM of the day of operation) while obeying to system constraints:

$$
\boldsymbol{i}^{o}=\underset{\boldsymbol{i} \in \theta}{\arg \min } \sum_{t=0}^{N-1}\left(\mathrm{SOC}_{t}-0.5\right)^{2}
$$

subject to

$$
\begin{array}{ll}
v_{t}=f(\boldsymbol{i}) & t=0, \ldots, N-1 \\
\mathrm{SOC}_{t}=g(\boldsymbol{i}) & t=0, \ldots, N-1 \\
B_{t}=v_{t} \cdot i_{t} & t=0, \ldots, N-1 \\
0 \leq \mathrm{SOC}_{t} \leq 1 & t=0, \ldots, N-1 \\
\left|\frac{B_{t}}{B_{\mathrm{nom}}}\right| \leq 1 & t=0, \ldots, N-1 \\
\frac{B_{t}+\widehat{L}_{t}}{P_{\text {nom }}} \leq 1 & t=0, \ldots, N-1,
\end{array}
$$

where $v_{t}$ is the estimated battery voltage, $f$ and $g$ are the battery prediction models (described in Section III-A3), $B_{t}$ is the battery active power consumption as seen by the BESS converter (equal to the battery voltage times the respective current as a first approximation), $P_{\text {nom }}$ is the active power rating of the BESS converter and $L_{\text {nom }}$ is the substation transformer nominal power. The inequality constraints in (11)(13) are respectively to keep battery SOC, power and power transit at the substation transformer within their prescribed limits. At this stage, we are not considering reactive power. In a real operation context, the constraints in (12) and (13) should be formulated using apparent power ratings instead. In such a case, the reactive power set-point of a four quadrant BESS converter could be determined by the MPC to compensate, for example, for all the reactive power of the feeder.

Once the battery current profile has been determined using the optimization problem presented above, the battery power demand is finally determined as (neglecting the power loss introduced by the converter):

$$
\widehat{B}_{t}^{o}=i_{t}^{o} \cdot f\left(i^{o}, t\right), \quad t=0, \ldots, N-1 .
$$

It is to be noted that, since this optimization is performed at $11 \mathrm{PM}$, the battery SOC at $0 \mathrm{AM}$ is unknown, and its value might vary in the last hour of the current intra-day operation. In this case, we simply approximate the SOC at midnight with the one at $11 \mathrm{PM}$.

3) Battery models:

a) Battery voltage: The voltage and current of a battery composed of $b$ parallel branches of $s$ cells in series can be expressed as:

$$
\begin{aligned}
& v_{t}=s V_{t} \\
& i_{t}=b I_{t},
\end{aligned}
$$

where $V_{t}$ and $I_{t}$ are respectively the cell voltage and current at the discrete time interval $t$. In the following, we assume that all the cells composing the battery have identical characteristics. In addition, we neglect slow time dynamics introduced by electrolyte aging processes. The battery model used in this paper is the one proposed by Bahramipanah et al. in [9] which model the rate capacity and charge redistribution effect ${ }^{1}$, and it is hereafter reformulated to be included into the proposed optimizations. The model is for a Lithium Titanate cell with nominal capacity and voltage of $30 \mathrm{Ah}$ and $2.3 \mathrm{~V}$, respectively. The cell voltage circuit model is shown in Fig. 3 and consists of a TTC (two time constants) model with a parallel controlled current generator. The former element describes the charge redistribution effect happening at the nominal cell current, while the latter captures the voltage dynamics introduced by a generic charging/discharging current. The circuit in Fig. 3 is described by the following dynamic equations (discretized at

\footnotetext{
${ }^{1}$ Respectively, the effect for which the battery capacity decreases when discharged with large values of current and the fact that during a phase of rest after, for example, a charge cycle, the battery voltage increases due to the redistribution of the charge in the battery electrolyte
} 
sample time $T_{s}=20$ seconds).

$$
\begin{aligned}
& V_{t}=E+R_{0}\left(I_{t}-I_{g, t}\right)+U_{1, t}+U_{2, t} \\
& U_{1, t+1}=U_{1, t}+\frac{1}{C_{1}}\left(-\frac{U_{1, t}}{R_{1}}+I_{t}-I_{g, t}\right) T_{s} \\
& U_{2, t+1}=U_{2, t}+\frac{1}{C_{2}}\left(-\frac{U_{2, t}}{R_{2}}+I_{t}-I_{g, t}\right) T_{s},
\end{aligned}
$$

where $I_{t}$ is the current extracted from the cell, and $R_{0}, R_{1}, R_{2}, C_{1}, C_{2}$ are fitted parameters dependent on the cell SOC according to the piecewise linear relationship summarized in the look-up table in Table I. The controlled current generator $E$ in (17) is modelled with the following polynomial model:

$$
\begin{aligned}
E\left(\mathrm{SOC}_{t}\right) & =0.61 \cdot \mathrm{SOC}_{t}^{4}+0.84 \cdot \mathrm{SOC}_{t}^{3}+ \\
& -2.1 \cdot \mathrm{SOC}_{t}^{2}+1.55 \cdot \mathrm{SOC}_{t}+1.80
\end{aligned}
$$

The controlled current source $I_{g, t}$ in (18) and (19) is as:

$$
I_{g, t}=Q_{r}\left(\frac{q_{1}}{R_{1} C_{1}} e^{\frac{t \cdot T_{s}-t_{0}}{R_{1} C_{1}}}+\frac{q_{2}}{R_{2} C_{2}} e^{\frac{t \cdot T_{s}-t_{0}}{R_{2} C_{2}}}+\frac{q_{3}}{\tau_{3}} e^{\frac{t \cdot T_{s}-t_{0}}{\tau_{3}}}\right)
$$

where $q_{1}, q_{2}, q_{3}, \tau_{3}$ are fitted parameters (shown in Table II) and $Q_{r}$ is the difference in achieved charge between the case when the cell is charged at its nominal current (said $I_{\text {Nom }}$ ) and when with the actual cell current profile. It is determined as:

$$
Q_{r}=\left\{\begin{array}{ll}
I_{\mathrm{Nom}} \cdot T_{s}-\frac{T_{s}}{t-t_{0}} \sum_{j=t_{0}}^{t-1} I_{t}, & i>0 \\
-I_{\mathrm{Nom}} \cdot T_{s}-\frac{T_{s}}{t-t_{0}} \sum_{j=t_{0}}^{t-1} I_{t}, & i<0 \\
I_{\mathrm{Nom}}-Q_{r, \mathrm{prev}}, & i=0 .
\end{array},\right.
$$

where $Q_{r \text {,prev }}$ corresponds to the charge achieved in the last charge/discharge phase. The discrete time index $t_{0}$ in (21)-(22) corresponds to the time step when the cell current turns from charging to discharging and vice-versa, i.e.

$$
t_{0}=t, \quad \text { if } \operatorname{sign}(t-1) \neq \operatorname{sign}(t) .
$$

\begin{tabular}{|c|c|c|c|c|c|c|c|}
\hline \multicolumn{2}{|c|}{ SOC } & 0 & 0.2 & 0.4 & 0.6 & 0.8 & 1 \\
\hline \multirow{5}{*}{ 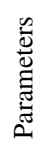 } & $R_{0}$ & 0.0030 & 0.0027 & 0.0018 & 0.0016 & 0.0022 & 0.0022 \\
\hline & $R_{1}$ & 0.0025 & 0.0019 & 0.0014 & 0.0014 & 0.0012 & 0.0019 \\
\hline & $R_{2}$ & 0.0025 & 0.0019 & 0.0014 & 0.0014 & 0.0013 & 0.0019 \\
\hline & $C_{1}$ & 10000 & 10730 & 10007 & 11488 & 10266 & 21289 \\
\hline & $C_{2}$ & 10000 & 10730 & 10007 & 11492 & 10266 & 21278 \\
\hline
\end{tabular}

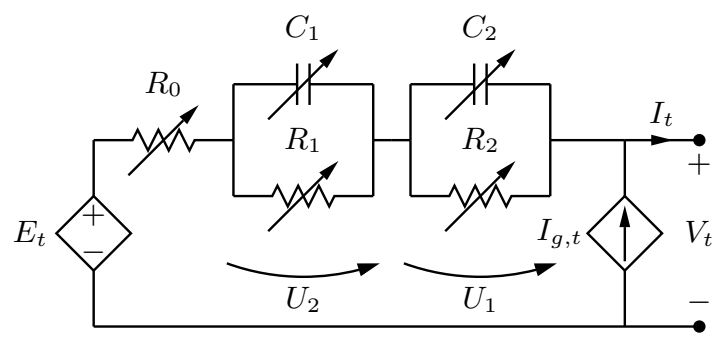

Fig. 3. The equivalent circuit of the battery charge redistribution effect model. The capacitance and resistance values are functions of the battery SOC.

TABLE I

LOOK-UP TABLE FOR THE CIRCUIT PARAMETERS VALUES.

Summarizing, the discrete dynamic equations in (17)-(19) together with (17)-(22) describe the cell voltage as a function
TABLE II

VALUES OF THE PARAMETERS OF THE MODEL IN (21).

\begin{tabular}{|c|c|c|c|}
\hline Parameter & $\begin{array}{c}\text { Charging } \\
\left(I_{t}<0\right)\end{array}$ & $\begin{array}{c}\text { Discharging } \\
\left(I_{t}>0\right)\end{array}$ & $\begin{array}{c}\text { Resting phase } \\
\left(I_{t}=0\right)\end{array}$ \\
\hline$q_{1}$ & 0.01 & 0.001 & 0.001 \\
$q_{2}$ & -0.03 & 0.03 & -0.03 \\
$q_{3}$ & 1.5 & 0.15 & 0.15 \\
$\tau_{3}$ & 900 & 900 & 900 \\
\hline
\end{tabular}

of the charging/discharging current $I_{t}$ accounting for the charge redistribution effect. Finally, the relationships in (15)(16) are used to model the complete battery bank.

b) Battery SOC: The battery SOC is often described as the integral over time of the charging/discharging current on the nominal capacity of the battery. However, this representation might be inaccurate as it does not account for the rate capacity effect. In this work, we utilize the following model, that is a discretized version of the model proposed in [10]:

$$
\operatorname{SOC}_{t+1}=\frac{C_{t}-\eta\left(i_{t}\right) i_{t} \Delta T}{Q\left(i_{t}\right)},
$$

where $C_{t_{0}}$ is the charge at time $t_{0}$ (in Coulomb), $\eta$ is the efficiency of the discharching process associated to the current $i_{t}$ (assumed equal to 1 as a first approximation), $\Delta T$ is the sampling time and $Q(I)$ is the battery capacity at the constant current discharge rate $I$ (information normally provided by the battery manufacturer). The coefficients $\eta$ and $Q$ are normally dependent also on the battery cells temperature. However, in this paper we have assumed the battery operating at a constant battery temperature.

\section{B. Intra-day operation}

1) Receding horizon MPC: At the beginning of the day of operation, the distribution feeder is dispatched using the 5minute advertised power consumption profile. The objective of the intra-day operation is to control the BESS to compensate for deviations from the advertised power consumption profile, that are likely to occur due to forecasting errors (see Section III-A1). The BESS control strategy is realized by using MPC and accomplishes the following targets:

- tracking of the advertised power consumption profile;

- plan the operation of the battery (in terms of current) such as its available capacity is the largest when the power consumption forecast are likely to be wrong in order to have an increased amount of energy to compensate for energy mismatches. This is done by accounting for the battery charge redistribution effect, which is modelled as described above.

The BESS MPC is applied in a receding horizon fashion once per minute. More specifically, the 5-minute interval is splitted into five 1-minute subperiods, at the beginning of those the MPC is recomputed with updated information, and only the first portion of the optimal control trajectory is applied.

a) Tracking error: We denote with $\widetilde{P}_{t, j}^{*}, j=0, \ldots, 4$ the 1-minute advertised power consumption profile obtained by 
up-sampling the 5-minute advertised power consumption setpoint $P_{t}^{*}$ (using linear interpolation) and define the tracking error (updated at the beginning of each 1-minute subperiod) as:

$$
\begin{aligned}
& e_{t, 0}=\widetilde{P}_{t, 0}^{*}-L_{t, 0} \\
& e_{t, j}=e_{t, 0}+\sum_{i=1}^{j} \widetilde{P}_{t, i}^{*}-\left(L_{t, i}+B_{t, i}\right), \quad j=1, \ldots, 4,
\end{aligned}
$$

where $L_{j}$ and $B_{j}$ are the actual measured average power absorptions of the feeder and battery. In words, the tracking error is the cumulative difference between the advertised power consumption and the total substation power transit. The situation and the input data of (25)-(26) is sketched in Fig. 4.

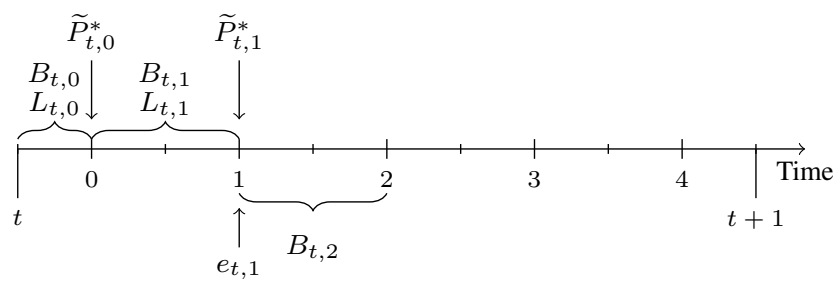

Fig. 4. The 5 -minute period $t$ is split into 5 subperiods $(0, \ldots, 4)$. In the example, we are at the beginning of subperiod 1: the tracking error $e_{t, 1}$ is computed as in (26) using the set-points $\widetilde{P}_{t, 0}^{*}, \widetilde{P}_{t, 1}^{*}$, battery power injections $B_{t, 0}, B_{t, 1}$ and feeder demand $L_{t, 0}, L_{t, 1}$. The first terms are obtained by up-sampling the advertised power consumption profile, while the last two are obtained averaging the respective measurements on the time intervals denoted by the curly braces. In the lower part, $B_{t, 2}$ is the actuated MPC action.

b) MPC formulation: At the beginning of each 1-minute interval (denoted by $t, j$, according to the notation introduced above), the MPC determines the 1-minute current trajectory for the next $L 5$-minute periods and is formulated as the following optimization problem:

$$
\boldsymbol{i}_{t, j}^{o}=\underset{\boldsymbol{i}_{t, j} \in \theta}{\arg \min }\left\{\alpha J_{0}+J_{1}\right\}
$$

where $\alpha$ is a weight coefficient, and the two terms $J_{0}$ and $J_{1}$, whose dependency from the battery current is omitted for simplifying the notation, are respectively as:

$$
\begin{aligned}
& J_{0}=\left(e_{t, j}-B_{t, j+1}\right)^{2} \\
& J_{1}=\sum_{h=t}^{t+L} \sum_{i=j}^{4}\left(\mathrm{SOC}_{h, i}-0.5\right)^{2}-w_{h, i} v_{h, i} .
\end{aligned}
$$

The weights $w_{h, i}$ in the expression above are obtained by mapping to the $[0,1]$ interval the sequence in (5) up-sampled to 1-minute resolution by linear interpolation. Formally, the weights for $t=0, \cdots, N-1, j=0, \cdots, 4$ are as:

$$
w_{t, j}=\frac{\widetilde{\widehat{V}}_{5 t+j}-\min \left(\mathcal{V}_{d+1 \mid d}\right)}{\max \left(\mathcal{V}_{d+1 \mid d}\right)-\min \left(\mathcal{V}_{d+1 \mid d}\right)} ;
$$

where $\widetilde{\widehat{V}}_{t}$ is an element of the 1-minute up-sampled version of the sequence $\mathcal{V}_{d+1 \mid d}$, and the operators max and min respectively denote the maximum and mininum value of the respective sequence. For example, $w_{h, i}$ equals 1 when the estimated variance of the power consumption prediction is the largest, and 0 when vice-versa. The optimization problem in (27) is subject to the following constraints:

$$
\begin{array}{ll}
v_{h, i}=f\left(\boldsymbol{i}_{t, j}\right) & h=t, \ldots, t+L, i=j, \ldots, 4 \\
\operatorname{SOC}_{h, i}=g\left(\boldsymbol{i}_{t, j}\right) & h=t, \ldots, t+L, i=j, \ldots, 4 \\
B_{h, i}=v_{h, i} \cdot i_{h, i} & h=t, \ldots, t+L, i=j, \ldots, 4 \\
0 \leq \operatorname{SOC}_{h, i} \leq 1 & h=t, \ldots, t+L, i=j, \ldots, 4 \\
\left|\frac{B_{h, i}}{B_{\text {nom }}}\right| \leq 1 & h=t, \ldots, t+L, i=j, \ldots, 4 \\
\frac{B_{h, i}+L_{h, i}}{P_{\text {nom }}} \leq 1 & h=t, \ldots, t+L, i=j, \ldots, 4 .
\end{array}
$$

The constraints (31)-(36) have the same meaning as for the previous optimization problem in Section III-A2. In words, the optimization problem seeks for the battery current profile that respects the operative constraints while tracking the advertised power consumption profile trough minimizing (28) and achieving large battery capacity (accounting for the charge redistribution) when the estimated forecast uncertainty is high, as in (29). Clearly, the operation of the MPC depends on the choice of the weight coefficient $\alpha$ in (27). Indeed, it determines the importance between terms $J_{0}$ and $J_{1}$ in the cost function. It is noteworthy that the optimization problem is nonconvex since the model $f$ in (31) necessary to determine (33) is nonlinear in the battery current.

\section{RESULTS AND DISCUSSION}

The framework is tested in simulation to verify its ability to dispatch the operation of a monitored distribution feeder of the EPFL campus [11]. The available power consumption measurements are split into daily sequences and used to determine the day-ahead power consumption forecast and the advertised power consumption profile. In the intra-day, the battery receding horizon MPC is applied to implement the dispatched consumption trajectory. The simulation conditions and the MPC parameters are in Table III.

TABLE III

SIMULATION SCENARIO SUMMARY.

\begin{tabular}{|l|c|l|}
\hline Description & Symbol & Value \\
\hline BESS capacity & - & $500 \mathrm{kWh}$ \\
Transformer power rating & - & $500 \mathrm{kVA}$ \\
Typical working day energy consumption & - & $4.80 \mathrm{MWh}$ \\
MPC look-ahead horizon & $L$ & 20 minutes \\
MPC cost function weight (Eq. (27)) & $\alpha$ & 1000 \\
\hline
\end{tabular}

Fig. 5 shows the advertised power consumption profile and the actual feeder power consumption. As expected, these two quantities differ due to inaccurate load forecast. The thicker line shows the implemented substation power transit (feeder power consumption plus the battery charging demand): the tracking action achieved by the MPC is well visible. Fig. 6 shows the simulated current and voltage profile of the battery cell model.

Table IV summarizes the simulation results for additional 5 daily scenarios. To prove the correct operation of the MPC, we introduce two metrics: the mean and scaled $L^{1}$ norm error, which are calculated for each simulation scenario 


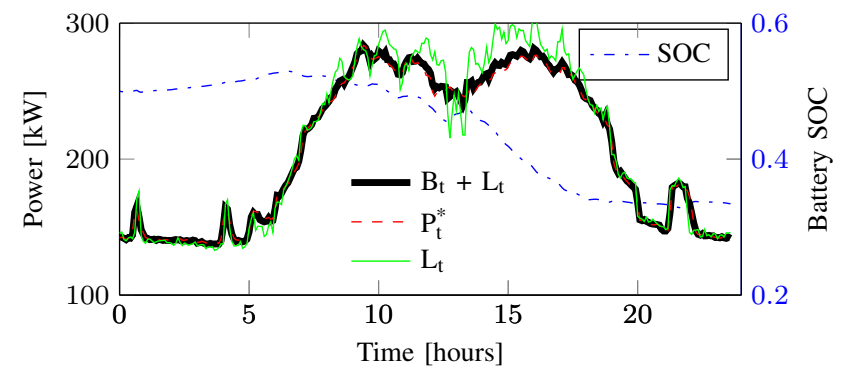

Fig. 5. The total power transit at the substation transformer $\left(B_{t}+L_{t}\right)$, the advertised power profile $\left(P_{t}^{*}\right)$ and the actual feeder power consumption $\left(L_{t}\right)$.

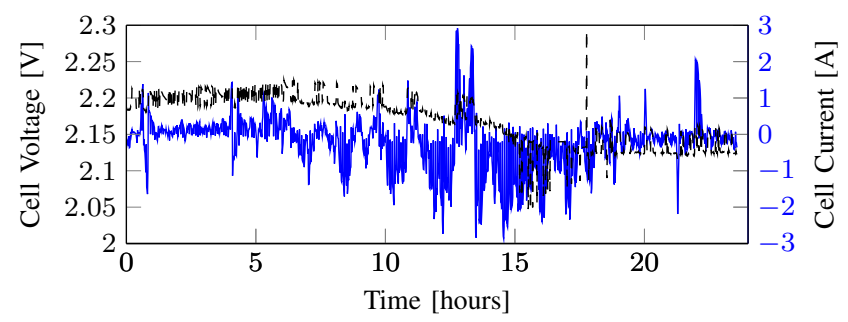

Fig. 6. The cell current (dashed black) and voltage (full blue).

to characterize the mismatches between i) the feeder power consumption realization and the advertised power consumption profile and ii) substation power transit after the MPC action and advertised power consumption profile. In the former case, the indicators measure the similarity between the advertised power consumption profile and the realization. In the latter, they measure the MPC ability to track the advertised power consumption profile. As visible from the numerical results, the MPC action is able to compensate for mismatches between the feeder power consumption and the advertised power consumption profile. The energy residual error is explained by the fact that the MPC does not currently track the advertised power consumption profile in the final 30 seconds of each 5-minutes period. This effect can be reduced by increasing the frequency of the MPC operation and also by implementing shorter term consumption forecast.

TABLE IV

SIMULATION RESULTS SUMMARY.

\begin{tabular}{|c|c|c|c|c|}
\hline \multirow{2}{*}{ Day } & \multicolumn{2}{|c|}{ Advertised profile vs Load } & \multicolumn{2}{c|}{ Power transit after MPC action } \\
\cline { 2 - 5 } & $\begin{array}{c}\text { Mean error } \\
(\mathrm{kW})\end{array}$ & $\begin{array}{c}\text { Daily energy } \\
\text { mismatch }(\mathrm{kWh})\end{array}$ & $\begin{array}{c}\text { Mean error } \\
(\mathrm{kW})\end{array}$ & $\begin{array}{c}\text { Daily energy } \\
\text { mismatch }(\mathrm{kWh})\end{array}$ \\
\hline 1 & 4.39 & 211.76 & 0.89 & 42.94 \\
2 & 12.68 & 392.79 & 3.08 & 91.65 \\
3 & -5.37 & 246.98 & -1.09 & 49.82 \\
4 & -4.77 & 181.33 & -0.95 & 37.02 \\
5 & -13.13 & 471.73 & -1.95 & 90.68 \\
\hline
\end{tabular}

\section{CONCLUSIONS AND FUtURE WORK}

Motivated by the target of reducing the amount of regulating power required to operate the grid (an enabling factor to achieve a larger proportion of production from renewables), we presented a framework to dispatch the operation of a distribution feeder using a battery energy storage system
(BESS). The framework is designed according to a day-ahead and intra-day structure to allow for its future integration with liberalized electricity market. In the day-ahead stage, the 5minute power consumption trajectory (said advertised power consumption profile) that the feeder should implement during the day of operation is determined using data-driven blackbox power consumption forecasting. In the intra-day stage, the feeder is dispatched according to the advertised power consumption profile. The problem of tracking the advertised power consumption profile is accomplished controlling the BESS power injection using receding horizon model predictive (MPC), which implements a state-of-the-art battery model to account for the battery charge redistribution and rate capacity effects. In comparison to conventional feedback control loop, the proposed strategy is characterized by larger flexibility as it allows for implementing the system operational constraints directly into the optimization problem while accounting for the battery behavior: it would be possible, for example, implementing battery aging models within the proposed framework. Future works will concern optimal battery sizing, integration of short term power consumption forecast in the MPC and experimental validation using a $0.5 \mathrm{MWh}$ Lithium Titanate BESS available at the EPFL-DESL facility.

\section{REFERENCES}

[1] B. Biegel, P. Andersen, J. Stoustrup, M. B. Madsen, L. H. Hansen, L. H Rasmussen et al., "Aggregation and control of flexible consumers-a real life demonstration," in Proceedings of the 19th IFAC World Congress, Cape Town, South Africa, 2014.

[2] S. Teleke, M. E. Baran, A. Q. Huang, S. Bhattacharya, and L. Anderson, "Control strategies for battery energy storage for wind farm dispatching," Energy Conversion, IEEE Transactions on, vol. 24, 2009.

[3] C. Hill, M. Such, D. Chen, J. Gonzalez, and W. Grady, "Battery energy storage for enabling integration of distributed solar power generation," IEEE Transactions on Smart Grid, vol. 3, no. 2, pp. 850-857, June 2012.

[4] H. W. Bindner, C. Krog Ekman, O. Gehrke, and F. R. Isleifsson, Characterization of vanadium flow battery. Danmarks Tekniske Universitet, Risø Nationallaboratoriet for Bæredygtig Energi, 2010.

[5] M. Zarghami, M. Vaziri, A. Rahimi, and S. Vadhva, "Applications of battery storage to improve performance of distribution systems," in Green Technologies Conference, 2013 IEEE, April 2013, pp. 345-350.

[6] M. Marinelli, F. Sossan, G. T. Costanzo, and H. W. Bindner, "Testing of a predictive control strategy for balancing renewable sources in a microgrid," IEEE Transactions on Sustainable Energy, 2014.

[7] L. Xie, Y. Gu, A. Eskandari, and M. Ehsani, "Fast mpc-based coordination of wind power and battery energy storage systems," Journal of Energy Engineering, vol. 138, no. 2, pp. 43-53, 2012.

[8] Ea Energy Analyses, "Activating electricity demand as regulating power," Tech. Rep., 2013. [Online]. Available: http://www.ea-energianalyse.dk/reports/1027_flexpower_ activating_electricity_demand_as_regulating_power.pdf

[9] M. Bahramipanah, D. Torregrossa, R. Cherkaoui, and M. Paolone, "Enhanced electrical model of lithium-based batteries accounting the charge redistribution effect," in Power Systems Computation Conference (PSCC), 2014.

[10] B. Belvedere, M. Bianchi, A. Borghetti, C. A. Nucci, M. Paolone, and A. Peretto, "A microcontroller-based power management system for standalone microgrids with hybrid power supply," Sustainable Energy, IEEE Transactions on, vol. 3, no. 3, pp. 422-431, 2012.

[11] M. Pignati, M. Popovic, S. Barreto Andrade, R. Cherkaoui, D. Flores, J.-Y. Le Boudec, M. M. Maaz, M. Paolone, P. Romano, S. Sarri, T. T. Tesfay, D.-C. Tomozei, and L. Zanni, "Real-Time State Estimation of the EPFL-Campus Medium-Voltage Grid by Using PMUs," in The Sixth Conference on Innovative Smart Grid Technologies (ISGT2015), 2014. 